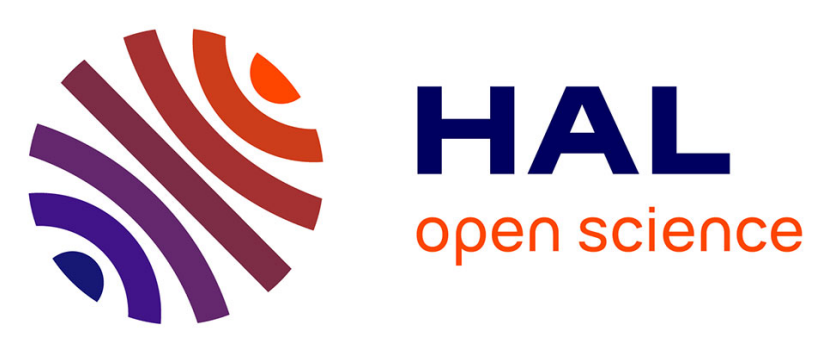

\title{
A resonant structure designed for probing the elastic properties of suspension and adherent cells in liquid environments
}

\author{
Denis Desmaële, Mehdi Boukallel, Stéphane Régnier
}

\section{- To cite this version:}

Denis Desmaële, Mehdi Boukallel, Stéphane Régnier. A resonant structure designed for probing the elastic properties of suspension and adherent cells in liquid environments. Journal of Micromechanics and Microengineering, 2012, 22, pp.115033. 10.1088/0960-1317/22/11/115033 . hal-00745077

\section{HAL Id: hal-00745077 \\ https://hal.science/hal-00745077}

Submitted on 24 Oct 2012

HAL is a multi-disciplinary open access archive for the deposit and dissemination of scientific research documents, whether they are published or not. The documents may come from teaching and research institutions in France or abroad, or from public or private research centers.
L'archive ouverte pluridisciplinaire HAL, est destinée au dépôt et à la diffusion de documents scientifiques de niveau recherche, publiés ou non, émanant des établissements d'enseignement et de recherche français ou étrangers, des laboratoires publics ou privés. 


\title{
A resonant structure designed for probing the elastic properties of suspension and adherent cells in liquid environmentsł
}

\author{
D Desmaële ${ }^{1,2}, \mathbf{M}$ Boukallel $^{1}$ and S Régnier ${ }^{2}$ \\ ${ }^{1}$ CEA, LIST, Sensorial and Ambient Interfaces Laboratory, 91191 Gif-sur-Yvette \\ Cedex, France \\ 2 Institut des Systèmes Intelligents et de Robotique, Université Pierre et Marie Curie, \\ CNRS - UMR 7222, 4 Place Jussieu, 75252 Paris Cedex 05, France \\ E-mail: denis.desmaele@etu.upmc.fr, mehdi.boukallel@cea.fr, \\ stephane.regnier@upmc.fr
}

\begin{abstract}
.
This paper presents a novel force sensitive structure exploiting a dynamic mode for probing the elastic properties of living cells. A key feature of this structure is the possibility to conduct measurements in liquid environments while keeping high dynamic performances. The structure indeed provides a steady area that can be adapted so that suspension or adherent cells can be placed in culture medium. The steady area is also connected to two adjacent beam resonators. Because these resonators never need to be immersed into the culture medium during measurements, forces applied to cells can be estimated with a high sensitivity via frequency shifts. In this paper, we conduct an extensive theoretical analysis to investigate the nonlinear effects of large static predeflections on the dynamic behavior of the structure. As a proof of concept, we also report the fabrication, characterization and calibration of a first prototype intended to deal with suspension cells with a diameter ranging from 100 to $500 \mu \mathrm{m}$. This prototype currently offers a quality factor of 700 and a force sensitivity of $\sim 2.6 \mathrm{~Hz} / \mathrm{mN}$. We also demonstrate that the prototype is capable of measuring the elastic modulus of biological samples in a rapid and sufficiently accurate manner without the need of a descriptive model.
\end{abstract}

PACS numbers: 87.85.fk, 87.17.Rt, 46.70.De, 62.40.+i

$\ddagger$ The final version of this paper has been published in Journal of Micromechanics and Microengineering by IOP Publishing Ltd and is available at http://iopscience.iop.org/0960-1317/22/11/115033/ (doi: 10.1088/0960-1317/22/11/115033). Please, cite this article as:

J. Micromech. Microeng. 22 (2012) 115033. 


\section{Introduction}

Measuring the elastic properties of individual living cells turns out to be of increasing interest. In particular, scientific evidences have revealed connections between alterations in the elastic modulus (i.e., Young's modulus) of single cells and pathophysiological states [1]. For instance, a dramatically reduced Young's modulus is a characteristic feature of cancerous cells $[2,3,4,5,6,7]$. By contrast, red blood cells infected by malaria or sickle cell disease have a significantly higher Young's modulus than their healthy counterparts $[8,9]$.

The elastic modulus of cells hence appears as a meaningful marker to differentiate pathogenic cells and healthy cells. For diagnostic purposes, knowing the Young's modulus of cells may hence help to detect the presence of cancer as well as other cellbased degenerative diseases at earlier stages. Furthermore, elasticity measurements also have the potential to disclose the specific effects of pharmaceuticals at the cellular level $[10,11,12]$. Therefore, cell elasticity measurements may also prove advantageous in drug development.

Among the large variety of macro and microscale devices that have been developed to investigate various mechanical aspects of living cells (see $[13,14,15,16,17]$ and references therein), the atomic force microscope (AFM) probably remains the most widespread tool for quantifying the Young's modulus of different types of cells. By way of example, results published in $[2,3,4,5,6,7,8,9]$ were all derived from measures obtained with AFM cantilevers.

Notwithstanding indisputable advantages, using an AFM in order to evaluate the Young's modulus of a living cell remains a delicate, time-consuming task. Basically, the extremity of the AFM cantilever must be cautiously and precisely positioned so that it can be used to indent or compress the cell. Then, a force-deformation curve must be acquired. Conventionally, a laser that reflects off the back surface of the cantilever onto a position sensitive photodiode is used to detect the contact point with the cell and to monitor the static deflection of the cantilever. However, if the cells are maintained in a culture medium during measurements, the alignment of the laser beam may become delicate. Moreover, in the presence of aqueous solutions, capillary meniscuses arise at the air-liquid interface when the AFM cantilever is immersed and removed. The compliant cantilever must then withstand large capillary forces that may engender measurement artifacts. Alternatively, an optical microscope and a video camera can be utilized [18]. The resolution is however limited by the optical components of the microscope and measurement uncertainties can occur. Furthermore, such vision-based techniques solely prove to be suitable for suspension cells with a spherical shape (e.g., red blood cells).

This paper introduces a novel force sensitive structure that has been designed with 
the aim to bring new solutions to these problems. In particular, the structure exploits a dynamic mode to quantify the Young's modulus of both suspension and adherent cells so that valuable information on the cell state can be obtained in a simple and sufficiently rapid manner for diagnosis applications.

\section{Overall description of the structure}

\subsection{Concept}

Structures exploiting resonance phenomena can outperform equivalent structures operated in a static mode [19]. Despite this potential advantage, resonant cantilevers are rarely used for determining the Young's modulus of living cells. Indeed, a major issue is that dynamic performances of AFM cantilevers are dramatically deteriorated as soon as they are surrounded by a viscous medium. Thereby, the quality factor of typical resonant AFM cantilevers immersed in liquids rarely exceeds 10-30 [20, 21].

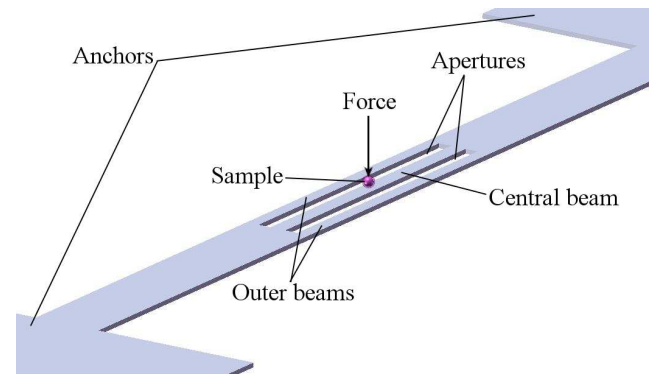

(a)

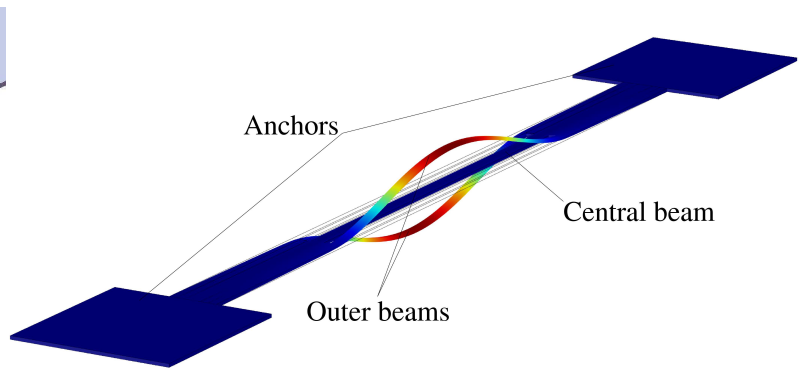

(b)

Figure 1. (a) Three dimensional schematic of the structure illustrated with a suspension cell (trapping system not represented). (b) FEA showing the antisymmetrical vibration mode exploited to extract the elastic properties of a biological sample: the two outer beams oscillate in antiphase whereas the third central beam remains immovable.

To extract the Young's modulus of a single living cell while taking full benefit of a dynamic mode, even in the presence of a liquid environment, we propose the suspended structure shown in Fig. 1(a). It consists of a planar structure clamped at its extremities. It also incorporates two rectangular apertures. Finite Element Analysis (FEA) conducted with COMSOL V.4 shows that if the structure is mechanically excited at the proper frequency, it can provide an antisymmetrical vibration mode where the two outer beams oscillate in antiphase. In the mean time, the third central beam remains immovable, as depicted in Fig. 1(b).

\subsection{Sensing principle}

Measuring the force applied to a cell is a prerequisite to extract its Young's modulus. Conceptually, if a static force normal to the structure's plane is applied to the half span of the central beam, the whole structure deflects. Therefore, the outer beams are 
forced to deflect as well. When the outer beams oscillate as illustrated in Fig. 1(b), interactions between static and dynamic behaviors occur. Thereby, the static deflection imposed by the normal force affects their initial resonance frequency. In fact, even slight static pre-deflection of beam resonators may significantly impact their dynamics [22]. Accordingly, it is possible to recover the magnitude of the normal force applied upon the central beam by monitoring frequency shifts of the outer beams, as it is demonstrated in Section 3.

\subsection{Key features}

The balanced mode of vibration shown in Fig. 1(b) is of particular interest for determining the Young's modulus of individual cells. Indeed, it enables high sensitivity rates offered by resonators without exposing cells to vibrations. Thereby, the area located at the half span of the central beam can be adapted to place suspension or adherent cells in liquids (see Fig. 2). A main advantage offered by such a configuration is that, unlike conventional AFM cantilevers, the oscillating outer beams do not need to be immersed in the liquid in order to measure a force applied to a cell. Therefore, major energy losses are avoided and high quality factor can be guaranteed. Because the outer beams always oscillate in air, potential difficulties related to laser alignments in liquids are eliminated and measurements based on vision techniques are not required. It is also worth noticing that since the central beam is inherently force sensitive, low-cost and commercially available microindenters can be used to apply forces on cells during experiments. Moreover, artifact measurements due to capillary effects are minimized since the microindenter is not directly used as a force sensor.

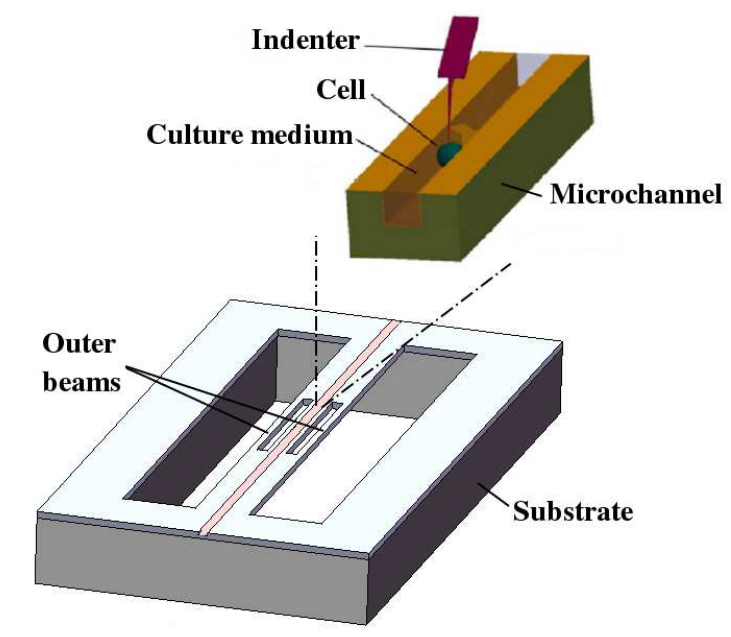

Figure 2. Top: Illustration of an open microfluidic channel for cell studies, as reported by Ryu et al. (adapted from [23, 24, 25]). Bottom: Conceptual view showing the structure equipped with a similar open microfluidic channel (fluid inlet and outlet not represented). 


\section{Theoretical analysis}

In this section, we demonstrate that the resonant structure can act as a force sensitive cell substrate. To predict frequency shifts engendered by a normal force $F$ applied to the central beam, static and dynamic behaviors are studied independently. We here mention that the experimental implementation of an open microchannel such as the one sketched in Fig. 2 is currently under investigation and is not addressed in this paper. Consequently, the presence of an open microchannel is not considered in the following analysis.

\subsection{Static behavior: large deflection of the structure}

A static analysis including nonlinear effects is first conducted in order to detail how the outer beams exactly behave during large deflections of the whole structure. Due to symmetry, and without loss of generality, our analysis can be limited to one fourth of the structure, as sketched in Fig. 3.

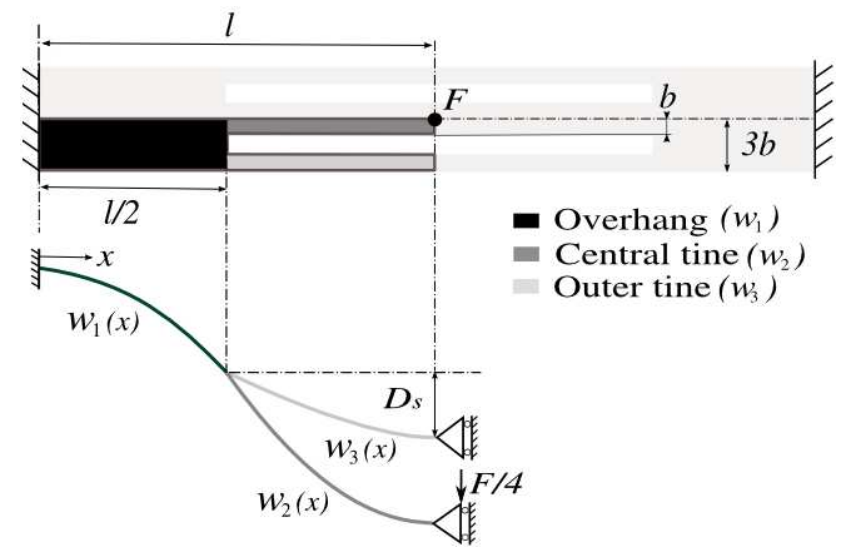

Figure 3. Top: for predicting the quasi-static deflection of the structure engendered by a normal force $F$, only the darker "tuning fork" (top view) is considered. Bottom: equivalent one-dimensional model (side view) of the colored beams (proportions exaggerated for illustration purposes).

This quarter structure, similar in shape to a tuning fork, is composed of three segments: the overhang (black), the central tine (dark gray), and the outer tine (light gray). All segments are assumed to satisfy Bernoulli's beam theory. One-dimensional coordinate functions are used to approximate the displacement field of each segment. To guarantee enough degrees of freedom, the flexural displacement $w_{1,2,3}(x)$ of each segment is modeled by third order polynomial expressions

$$
\left\{\begin{array}{l}
w_{1}(x)=a_{0}+a_{1} x+a_{2} x^{2}+a_{3} x^{3} \\
w_{2}(x)=a_{4}+a_{5} x+a_{6} x^{2}+a_{7} x^{3} \\
w_{3}(x)=a_{8}+a_{9} x+a_{10} x^{2}+a_{11} x^{3} .
\end{array}\right.
$$


The three polynomials in (1) are then used to calculate the total potential energy stored by the tuning fork during deflection

$$
U_{t f}=U_{b}+U_{s}
$$

where $U_{b}$ is the sum of strain energies developed by each segment during bending

$$
U_{b}=\sum_{i=1}^{3} \frac{1}{2} E I_{i} \int_{c_{i}}^{d_{i}}\left(\frac{d^{2} w_{i}}{d x^{2}}\right)^{2} d x .
$$

In (3) $c_{1}=0, d_{1}=l / 2, c_{2,3}=l / 2, d_{2,3}=l . I_{i}$ are the moments of inertia of the three segments with $I_{1}=b h^{3} / 4$ and $I_{2}=I_{3}=b h^{3} / 12, E$ represents the Young's modulus of the structure's material, the width $b$ and the length $l$ are defined in Fig. 3 and $h$ is the structure's thickness.

In addition, $U_{s}$ is the sum of energy contributions due to the midplane stretching of the segments that occurs during deflection

$$
U_{s}=\sum_{j=1}^{3} \frac{E A_{j}}{4 l}\left[\int_{c_{j}}^{d_{j}}\left(\frac{d w_{j}}{d x}\right)^{2} d x\right]^{2}
$$

where $c_{1}=0, d_{1}=l / 2, c_{2,3}=l / 2$ and $d_{2,3}=l$. $A_{j}$ in (4) represents the cross section areas of the three segments with $A_{1}=3 b h$ and $A_{2,3}=b h$. The total potential energy function can hence be written as

$$
\Phi=U_{t f}-W
$$

where $W=F w_{2}(l)$ is the work done by the punctual force $F$.

Applying the principle of minimum potential energy, one sets for each unknown coefficient $a_{i}$

$$
\frac{\partial \Phi}{\partial a_{i}}=0
$$

The problem is then augmented with Lagrange multipliers by considering a set of constraints. To obtain satisfactory results, mechanical constraints do not need to be considered. Enforcing geometrical constraints is enough to provide an accurate solution. Assuming that the central tine and the outer tine are rigidly linked to the overhang at $x=l / 2$, and that the central and outer tines are terminated by sliding conditions (see Fig. 3), the following set of conditions can be enforced

$$
\begin{aligned}
& w_{1}(0)=\frac{d w_{1}}{d x}(0)=0 \\
& w_{1}(l / 2)=w_{2}(l / 2)=w_{3}(l / 2) \\
& \frac{d w_{1}}{d x}(l / 2)=\frac{d w_{2}}{d x}(l / 2)=\frac{d w_{3}}{d x}(l / 2) \\
& \frac{d w_{2}}{d x}(l)=\frac{d w_{3}}{d x}(l)=0 .
\end{aligned}
$$


The augmented system can then be numerically solved for different values of $F$. To compute such a system, an algorithm such as the one reported in [26] was used with the following numerical values: $l=12.5 \mathrm{~mm}, b=0.25 \mathrm{~mm}, h=0.1 \mathrm{~mm}, E=212 \mathrm{GPa}$.

To validate this modeling approach, FEA was used as a reference tool. COMSOL simulations were conducted in the nonlinear deflection mode with the values of $l, b, h$ and $E$ aforementioned. We also specified a density of $8030 \mathrm{~kg} \mathrm{~m}^{-3}$ and a Poisson's ratio of 0.29 for the structure's material. These values were selected in accordance with the material utilized for the prototype of Section 4. For a point force $F=260 \mathrm{mN}$ applied at the half span of the central beam, Fig. 4 shows the deflection profile of the structure. Analytical results are in good agreement with FEA simulations and permit to predict the deflection of the structure accurately. It can also be seen that the deflection amplitude of the outer beam is smaller than the deflection of the central beam.

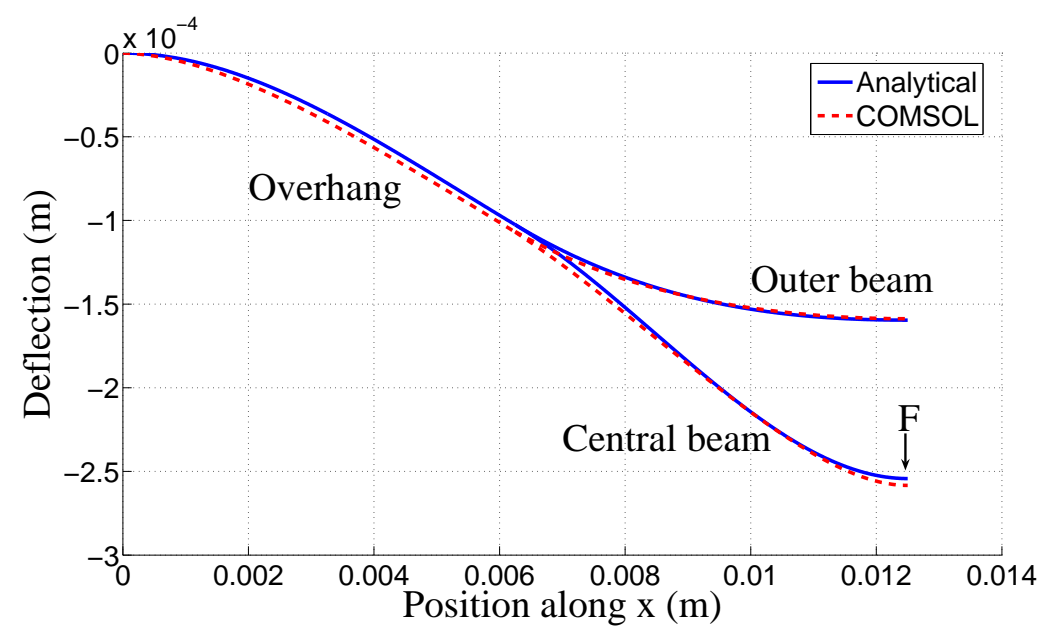

Figure 4. Deflection profile and deflection amplitude when a normal force $F=260 \mathrm{mN}$ is applied at the half span of the central beam.

This intuitive result is also confirmed by Fig. 5 which compares the deflection amplitude of both beams as a function of the force $F$ applied. Analytical and FEA results are again in accordance. Figure 5 also clearly illustrates that the stretching (i.e., nonlinear) effect progressively dominates as the beams deflection increases. Thereby, for deflections above $\sim 50 \%$ of the structure's thickness, the structure becomes clearly stiffer.

\subsection{Dynamic analysis: effects of a static predeflection on the oscillation of the outer} beams

We now demonstrate that, if the force $F$ is applied while the outer beams oscillate as shown in Fig. 1(b), the variations of deflection imposed by $F$ actually impact the initial resonance frequency of the outer beams. To demonstrate such a coupling between static and dynamic behaviors, a symmetric structure again simplifies the analysis. Indeed, 


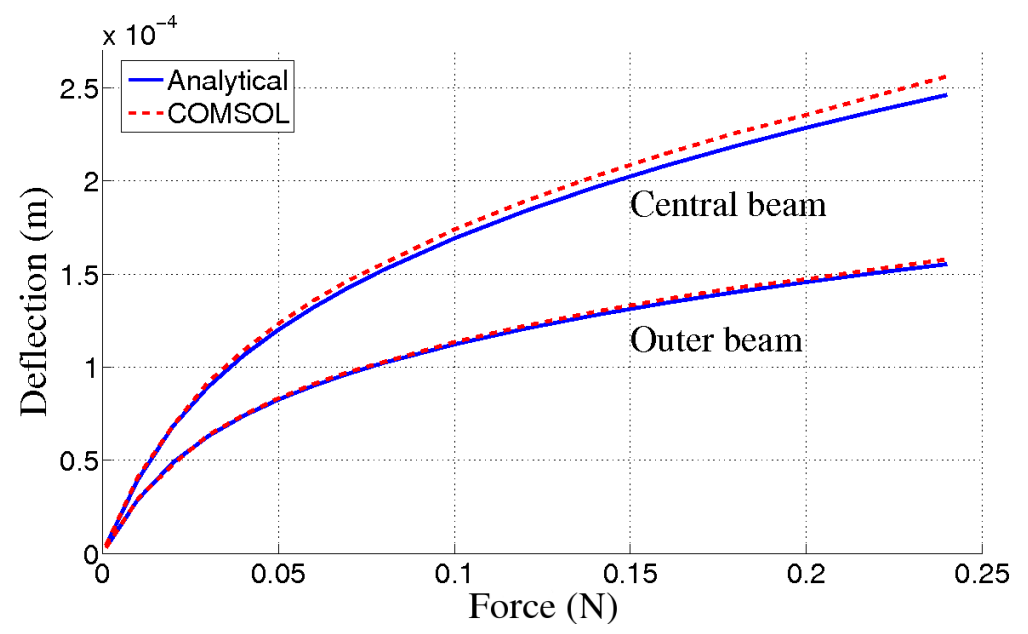

Figure 5. Half span deflections of the central beam and one outer beam as a function of the force $F$ applied to the central beam. Due to symmetry, the second outer beam deflects exactly in the same manner.

valuable insights into the structure's dynamics can be grasped by restricting the analysis to a single outer beam (see Fig. 6).

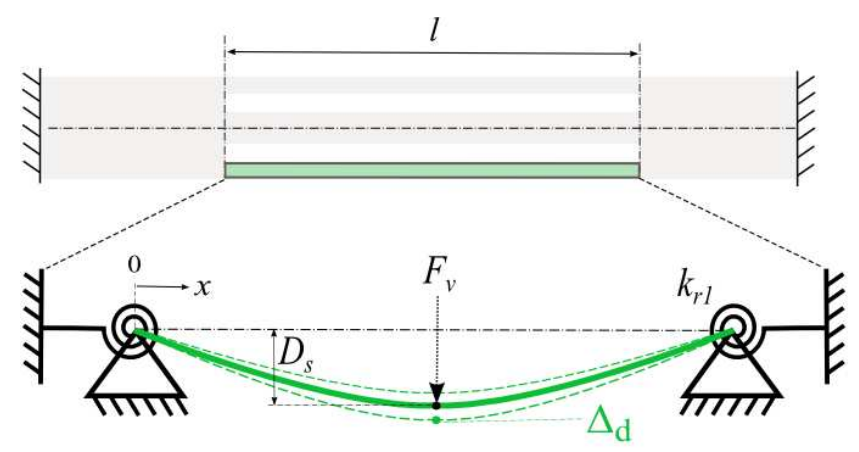

Figure 6. For predicting frequency shifts, only one outer beam is considered. Vibrations (dashed green lines) take place around an equilibrium position (thick green line). The curved shape is indirectly engendered by static deflections (proportions not to scale)

To a first approximation, one can choose to model the outer beam as a hingedhinged beam terminated by two rotational springs of stiffness $k_{r 1}$ (see Fig. 6). The presence of rotational springs is justified by the facts that extremities of the outer beam are attached to the overhangs. Intuitively, it can be foreseen that, although the overhangs can be considered as axially immovable ends, they should not act as ideal clamping supports. In the literature, elastic rotational springs are often used to model such a flexibility $[27,28,29]$.

Again, energy approaches can be exploited to conveniently handle the configuration 
of Fig. 6. Because the outer beam is terminated by rotational springs attached to pinned ends supports, it is conventional to assume a displacement function in the form of a sine

$$
w(x)=D_{s} \sin \left(\frac{\pi x}{l}\right)
$$

where $D_{s}$ is the midspan deflection of the outer beam.

$D_{s}$ is related to the force $F$ applied upon the central steady beam, and can be estimated from the previous static analysis (see Fig.3)

$$
D_{s}=w_{3}(l)-w_{1}(l / 2) .
$$

Conceptually, it is however more convenient to consider that $D_{s}$ is rather due to an unknown equivalent virtual force $F_{v}$ (see Fig. 6). With an energy approach, a relationship between $D_{s}$ and $F_{v}$ can be derived. Considering that $F_{v}$ is applied at the beam midspan, the potential energy stored by the beam is the sum of the following contributions

$$
U_{T}=U_{b}+U_{s}+U_{r s} .
$$

In (13) $U_{b}$ is the bending energy of the beam

$$
U_{b}=\frac{E I}{2} \int_{0}^{l}\left(\frac{d^{2} w}{d x^{2}}\right)^{2} d x
$$

$U_{s}$ is the energy developed during the midplane stretching of the beam

$$
U_{s}=\frac{E A}{8 l}\left[\int_{0}^{l}\left(\frac{d w}{d x}\right)^{2} d x\right]^{2}
$$

and $U_{r s}$ is the energy stored by the two rotational springs of stiffness $k_{r 1}$

$$
U_{r s}=\frac{k_{r_{1}}}{2} \theta_{0}^{2}+\frac{k_{r_{1}}}{2} \theta_{l}^{2}
$$

where $\theta_{0}$ and $\theta_{l}$ are the slopes $\frac{d w}{d x}$ evaluated at $x=0$ and $x=l$, respectively.

Considering that the work done by $F_{v}$ is $W_{1}=F_{v} w(l / 2)$, the total potential energy function $\Phi_{1}$ is

$$
\Phi_{1}=-F_{v} D_{s}+\left[\frac{\pi^{2} k_{r_{1}}}{l^{2}}+\frac{E b h^{3} \pi^{4}}{48 l^{3}}\right] D_{s}^{2}+\frac{E b h \pi^{4}}{32 l^{3}} D_{s}^{4} .
$$

Minimizing (17) with respect to $D_{s}$ yields a cubic force-centered-deflection law

$$
F_{v}=k_{1} D_{s}+k_{3} D_{s}^{3}
$$

where

$$
k_{1}=\frac{E b h^{3} \pi^{4}}{24 l^{3}}+\frac{2 k_{r_{1}} \pi^{2}}{l^{2}} \quad \text { and } \quad k_{3}=\frac{E b h \pi^{4}}{8 l^{3}} .
$$

In (19), $k_{1}$ and $k_{3}$ are linear and nonlinear spring constants, respectively. 
As in [30], it can now be assumed that small deflections of the beam about a mean deflection $D_{s}$ can be described approximately by a single stiffness value. An effective spring constant is found by deflecting the beam from its equilibrium position by an arbitrary amount $\Delta_{d}$ (see Fig. 6), so that

$$
F_{v}=k_{1}\left(D_{s}+\Delta_{d}\right)+k_{3}\left(D_{s}+\Delta_{d}\right)^{3} .
$$

Considering that vibration amplitudes $\Delta_{d}$ are sufficiently small, terms proportional to $\Delta_{d}^{2}$ and $\Delta_{d}^{3}$ in (20) can be neglected. Then, an equivalent stiffness of the outer beam may be approximated by

$$
k_{e q}=\frac{d F_{v}}{d \Delta_{d}} \approx k_{1}+3 k_{3} D_{s}^{2}
$$

As often in structures involving coupled beams, vibrating beams can be modeled to a first approximation as one degree of freedom oscillators (e.g., [31, 32]). If the single outer beam is assumed to oscillate as an undamped lumped-parameter system, the static deflection $D_{s}$ affects its natural frequency as follows

$$
\frac{f}{f_{0}} \approx\left[1+\frac{3 k_{3}}{k_{1}} D_{s}^{2}\right]^{1 / 2} .
$$

As previously, the numerical values $E=212 \mathrm{GPa}, b=0.25 \mathrm{~mm}, l=12.5$ and $h=0.1 \mathrm{~mm}$ were used to compute $(22)$. An order of magnitude for the rotational stiffness $k_{r 1}$ was also found by using [33]

$$
k_{r 1}=\frac{4 E b h^{3}}{3 l} \text {. }
$$

Equation (23) gives $k_{r 1} \approx 5.6 \times 10^{-3} \mathrm{Nm} / \mathrm{rd}$. Nonetheless, $(23)$ is originally intended to estimate the rotational spring constant of anchors for clamped-clamped beams. In our case, the outer beams are attached to the overhangs (see Fig. 3) which do no act as ideal clamps. Therefore, the value of $k_{r 1}$ was slightly adapted to fit prestressed modal analyses conducted with COMSOL. For $k_{r 1}=3.45 \times 10^{-3} \mathrm{Nm} / \mathrm{rd}$, Fig. 7 proves that (22) accurately predicts the amount of frequency change engendered by a force $F$ applied to the central beam.

Alternatively, and because displacements of the central beam can also be predicted with respect to the force $F$ applied, the amount of frequency change can also be estimated as a function of a displacement imposed to the central beam (see Fig. 8). It is of interest to note that in both cases, quasi linear regions are predicted around points $P$. In particular, a force sensitivity of $2.91 \mathrm{~Hz} / \mathrm{mN}$ is predicted if the force applied to the central beam exceeds $100 \mathrm{mN}$ (see Fig. 7).

\section{Experiments}

To demonstrate the possibility to extract the Young's modulus of living cells with the structure, we fabricated a first prototype intended to deal with suspension cells whose diameter approximately ranges from 100 to $500 \mu \mathrm{m}$. This monolithic prototype was 


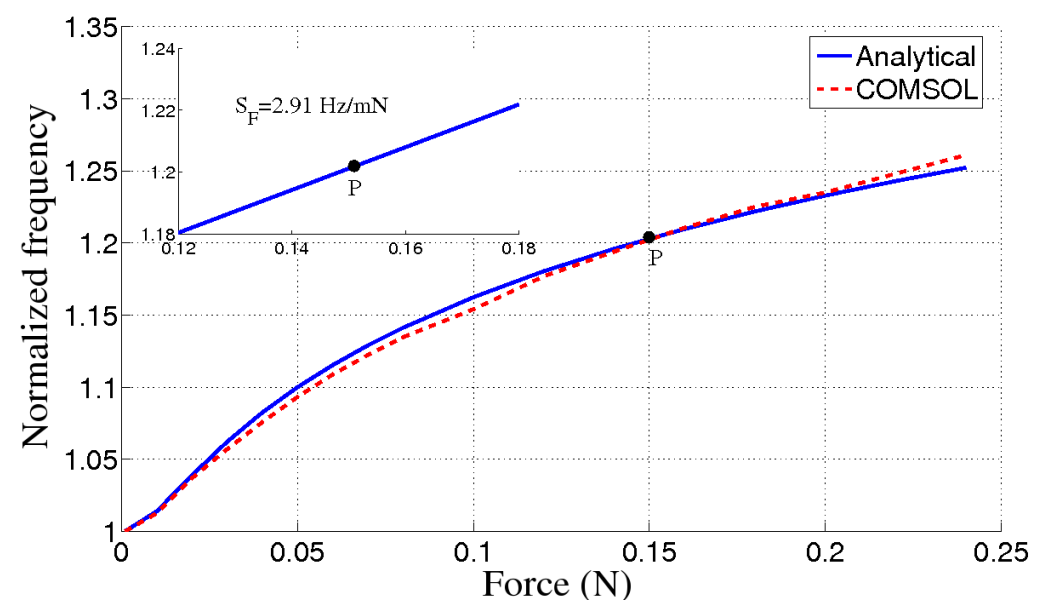

Figure 7. Evolution of the resonance frequency of the outer beams when a vertical force $F$ is applied upon the half span of the central beam.

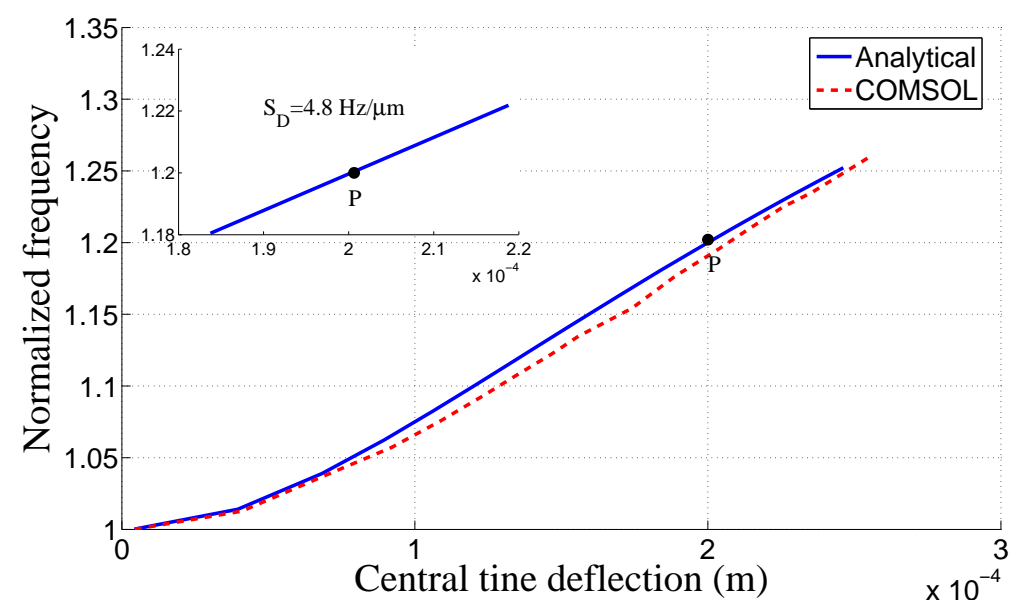

Figure 8. Evolution of the resonance frequency of the outer beams when a vertical displacement is imposed upon the half span of the central beam.

fabricated from a single sheet of biocompatible stainless steel. All dimensions are those used for the theoretical analysis. Considering the total length of the prototype $(25 \mathrm{~mm})$, precision wire cut electric discharge (EDM) machining was favored for rapid prototyping. Wire cut EDM, however, did not permit to implement an open microchannel.

Mechanical excitation was provided by a $3 \mathrm{~mm}$ long, $2 \mathrm{~mm}$ wide and $200 \mu \mathrm{m}$ thick piezoelectric (PZT) element (Physik Instrumente PIC151). This PZT element was bonded onto the prototype with conductive paste and driven by an AC signal with a function generator (Agilent 33120A) connected to a laboratory power amplifier (Newtons4th LPA400).

During experiments, the prototype was suspended between two clamps. One of the 
clamps was fixed to a manual micropositioning stage that allowed horizontal translations along the $y$ direction. To ensure a firm attachment of the prototype and to avoid slipping, the clamps were tightened with screws. The whole setup (see Fig. 9) was mounted on a pneumatic antivibration isolation table to minimize the presence of external disturbances. To measure small static deflection as well as beam oscillations, we do not use a laser coupled to a sensitive photodiode. Instead, we used a home made optical fiber displacement probe. This probe was constituted of two step index multimode fibers having a diameter of $50 \mu \mathrm{m}$ at their extremity. Such dimensions offered a compact sensing head that could also be implemented in a future microfabricated version of the structure. Further details about this fiber displacement probe can be found in [34].

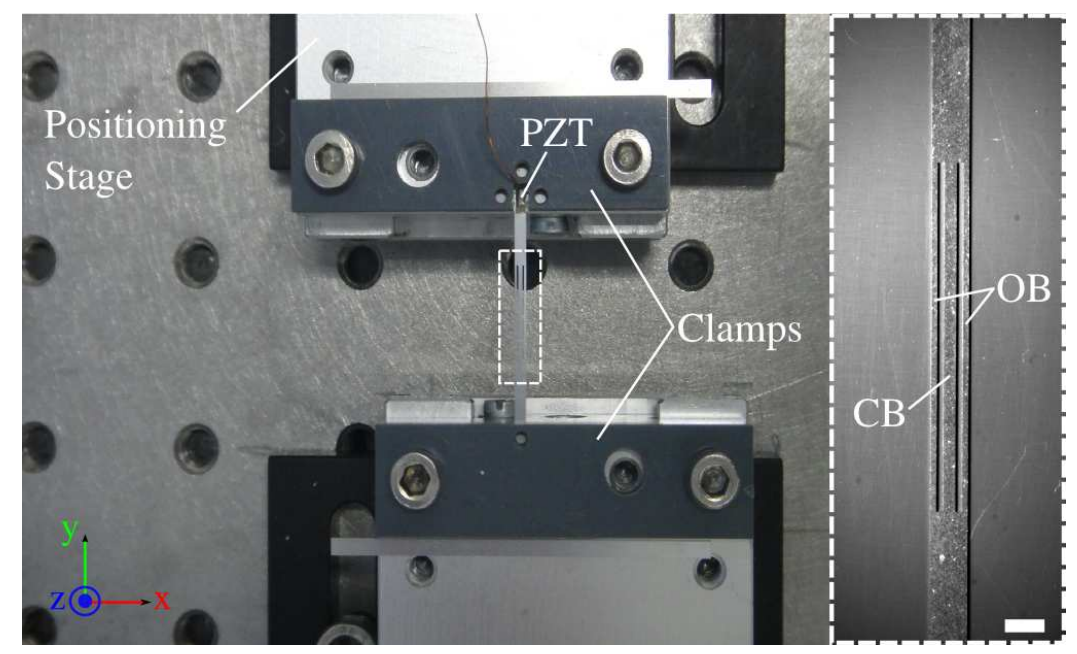

Figure 9. Top view of the prototype and experimental arrangement. The inset provides a microscope view of the area indicated by dashed lines. CB and OB stand for central beam and outer beams, respectively. Scale bar equals $1.5 \mathrm{~mm}$.

\subsection{Evaluation of static deflections}

Experiments were first carried out to investigate the static deflection of the prototype. To bend the structure, an indenter terminated by a metal bead with a diameter of $500 \mu \mathrm{m}$ was placed beneath the central beam. The stiffness of the metal bead was much higher than the stiffness of the structure. With a micropositioning stage (Physik Instrumente M112-1DG), incremental motions of $10 \mu \mathrm{m}$ along the $z$ direction were imposed to the central beam. Considering the prototype symmetry, deflection data were acquired only for one outer beam.

Fig. 10 demonstrates that the prototype deflected as expected during experiments, even though the deflection of the outer beams tends to be $12 \%$ overestimated by theory when the deflection of the central beam is above $30 \mu \mathrm{m}$. Several uncertainties sources could explain this slight estimation error (e.g., values of Young's modulus and Poisson's 


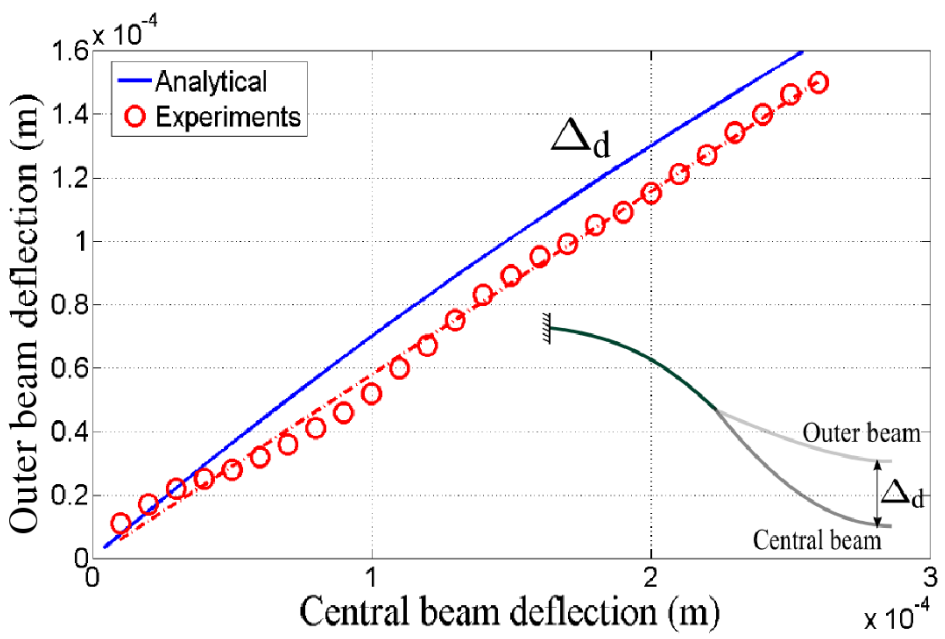

Figure 10. Difference of static deflection $\Delta_{d}$ between the central and the outer beams. Solid line is plotted thanks to the curves from Fig. 5. Circles represent experimental data. Dashed line is a fitting curve.

ratio used for calculations; uncertainties related to the fabrication process, the clamping of the prototype, etc.).

\subsection{Evaluation of dynamic performances}

The dynamic behavior of the prototype was then explored. In a first step, the indenter was removed and no force was applied to the central beam. To actuate the prototype, the PZT element was driven with a sinusoidal voltage. The peak-peak amplitude of this sine signal after the power amplifier was $9 \mathrm{~V}$. The excitation frequency was then swept with the function generator in order to find the resonance modes of the prototype. As illustrated by Fig. 11(a), the antisymmetrical mode of interest was found at $3180 \mathrm{~Hz}$.

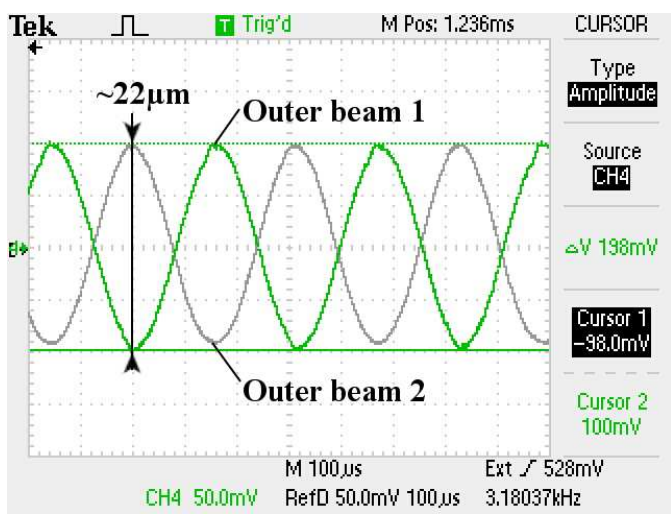

(a)

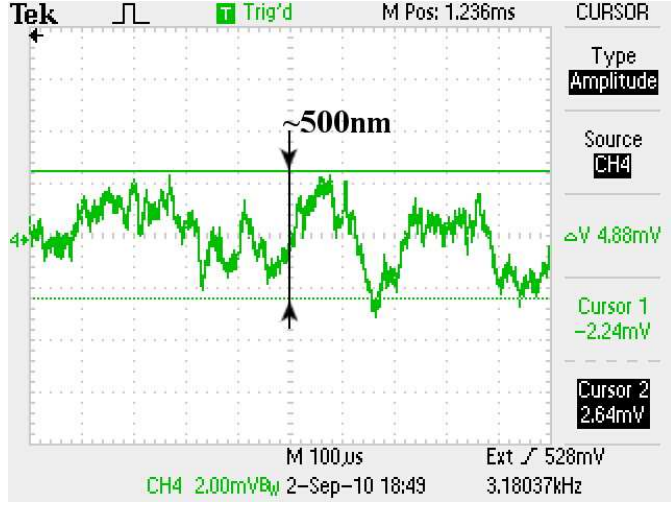

(b)

Figure 11. Oscilloscope screenshots showing: (a) The two outer beams oscillating in antiphase when the structure is driven at $3180 \mathrm{~Hz}$; (b) Vibrations measured at the half span of the central beam with no force applied. 
When the PZT element was driven with a voltage supply of $9 \mathrm{~V}$, the peak-peak oscillation amplitude of the outer beams was $22 \mu \mathrm{m}$. During oscillations of the outer beams, a lot of attention was paid to check if potential vibrations were transferred to the central beam. As a matter of fact, they were very limited. As shown in Fig. 11(b), they never exceeded $500 \mathrm{~nm}$, that is to say $2 \%$ of the oscillation amplitude of the outer beams. Droplets of water were also deposited upon the central beam with a micropipette. No particular effect on the droplets was observed. It was also confirmed that the presence of small amounts of liquids on the central beam did not alter the vibration mode.

In addition, we explored the frequency response of the antisymmetrical vibration mode. With the outer beams oscillating in air, a quality factor of 700 was obtained (see Fig. 12). This quality factor was not deteriorated when a liquid droplet was deposited on the central beam. This is a high value when compared to typical quality factors of AFM cantilevers in liquids (10-30).

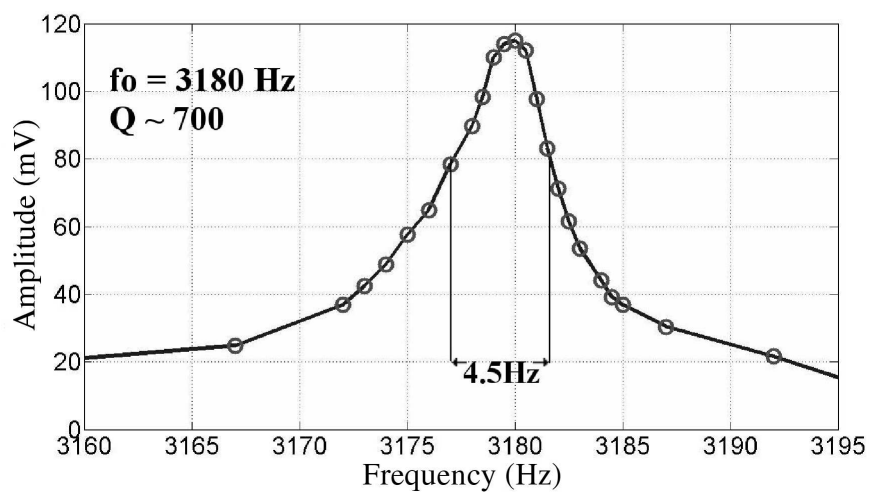

Figure 12. Experimental frequency response for the prototype of Fig. 9 driven around $3180 \mathrm{~Hz}$.

\subsection{Frequency variations induced by large displacements}

The coupling between static and dynamic behaviors was then investigated. To that end, oscillations of the outer beams were monitored while the central beam was bent with the indenter. For a total displacement of the indenter of $250 \mu \mathrm{m}$, the oscillation frequency of the outer beams evolved as shown in Fig. 13.

Contrary to theoretical predictions, it is experimentally observed that for small deflections of the central beam (i.e., deflections lower than $100 \mu \mathrm{m}$ ), the frequency of the outer beams actually decreases. Such a behavior has been reported for buckled and deflected beams subjected to axial loads [35, 36, 37, 38, 39]. Nevertheless, this decrease finally appears as a transition period. Indeed, once the stretching effect dominates at large deflections, a behavior very similar to the one initially predicted is retrieved. In particular, it is worth noticing that the curve in Fig. 13 offers a linear displacement 


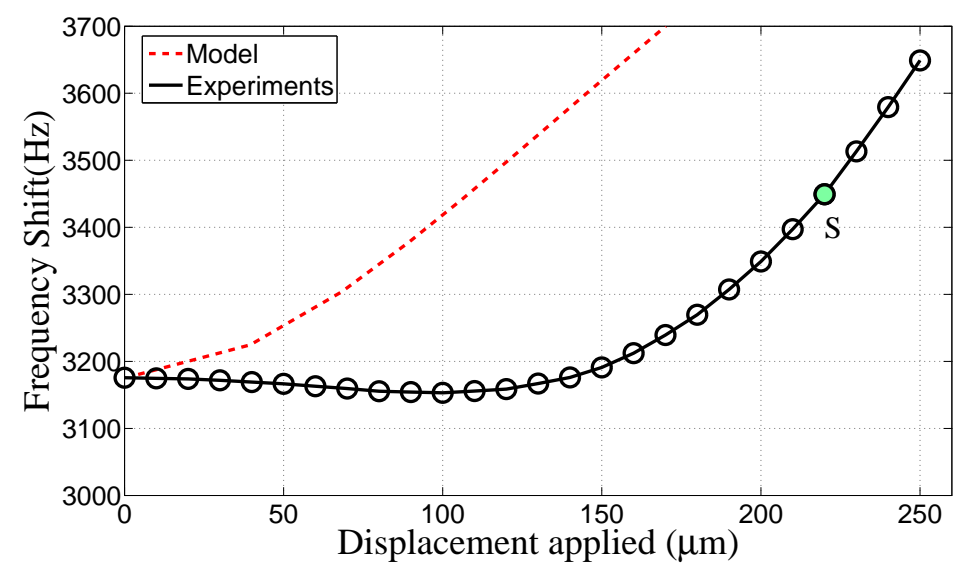

Figure 13. Frequency variations measured for a normal displacement applied to the half span of the central beam. Circles and solid line correspond to experimental data. Dashed line represents variations predicted by Fig. 8 for the same initial frequency.

sensitivity of $5.75 \mathrm{~Hz} / \mu \mathrm{m}$ around the point $S$. This displacement sensitivity is actually $\sim 17 \%$ better than the one expected initially by theory $(4.8 \mathrm{~Hz} / \mu \mathrm{m})$.

\subsection{Frequency variations induced by small forces}

From the analysis of Fig. 13, it is clear that the prototype provides a much better sensitivity when the central beam is largely bent. The central beam was hence maintained permanently curved (i.e., even with the indenter removed) to exploit the region around the point $S$. Obviously, this axial translation compressed the whole structure. As a result, the initial resonance of the antisymmetrical vibration mode decreased from $3180 \mathrm{~Hz}$ to $3080 \mathrm{~Hz}$. Nevertheless, no significant impact on the dynamic behavior of the prototype was noticed.

To estimate the force $F$ applied to the central beam during small displacement intervals, the force developed by our indenter was characterized. By pressing the indenter against a precision electric scale (Kern 440-33), a linear relationship was found (see Fig. 14(a)). Then, frequency variations with respect to the force generated by the indenter were measured. With the prototype predeflected, only a linear increase of the frequency was observed, as expected (see Fig. 14(b)). This linear increase corresponded to a force sensitivity of $2.56 \mathrm{~Hz} / \mathrm{mN}$. Although a predeflection of the whole structure was not included in our theoretical analysis, the force sensitivity measured experimentally was only $\sim 12 \%$ lower than expected.

\subsection{Direct extraction of the elastic modulus of a suspension cell}

With the suspended structure, the force applied to a cell can be measured. In addition, the cell deformation must also be determined. Conventionally, force-deformation information are then used in conjunction with Hertz theory to retrieve the Young's modulus 


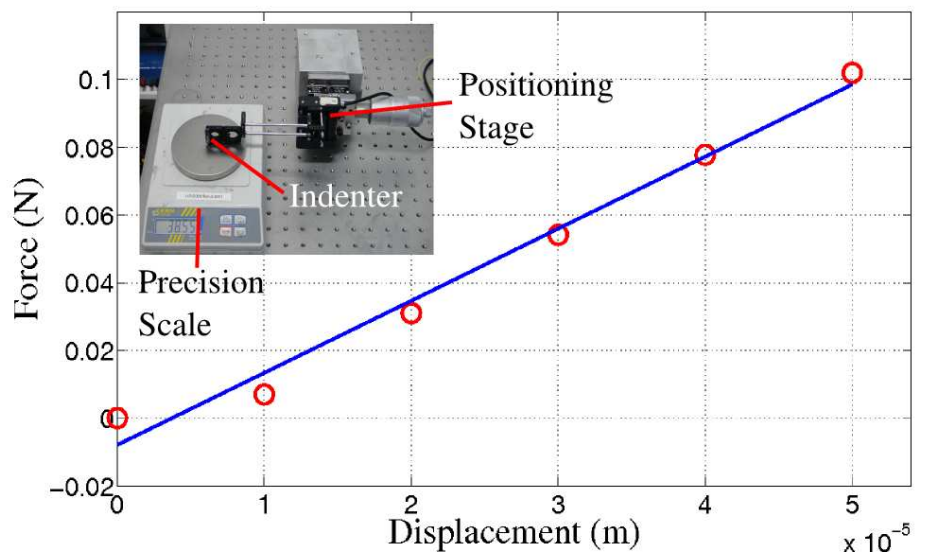

(a)

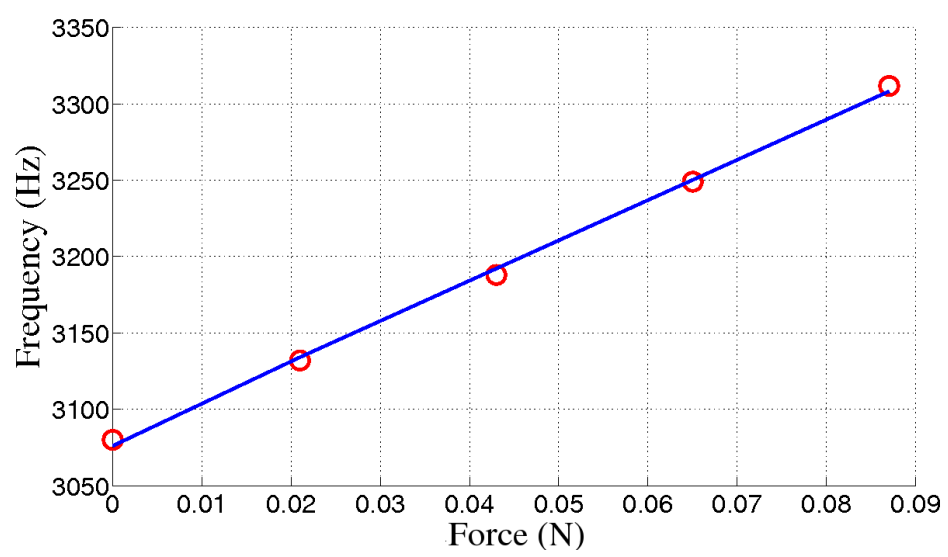

(b)

Figure 14. (a) Experimental force-displacement relationship obtained for small displacements of the indenter. (b) Lin ear frequency shift measured for the structure maintained slightly curved. Circles correspond to experimental data. Solid lines are fitting curves.

of the cell. Hertz theory, however, is subjected to a number of important assumptions. In particular, when dealing with a living cell, the condition of negligible force adhesion is not always fulfilled. Furthermore, for suspension cells, Hertz theory requires to know the radius of curvature of the cell to be probed. As an alternative, we preferred to calibrate our structure so that a sufficient estimation of the Young's modulus of the cell could be rapidly obtained without the use of a descriptive model. For calibration, two gel samples T7 and T5 were bought from the company Gelmec. They had a Young's modulus of $37.5 \mathrm{kPa}$ and $119 \mathrm{kPa}$, respectively.

The gel samples were manually prepared so that their size was as similar as possible to the size of the cells targeted. Thereby, efforts were made to obtain small cubic pieces whose edges measured approximately $500 \mu \mathrm{m}$. The gel samples were then cautiously placed on a flat indenter and compressed upon the central beam of the structure. To 
probe only the elastic properties of the gels, the amount of compression applied did not exceed $10 \%$ of their thickness [40]. Moreover, the velocity of the translation steps was kept very slow $(4 \mu \mathrm{m} / \mathrm{s})$ to minimize the occurrence of viscoelastic effects $[18,41]$.

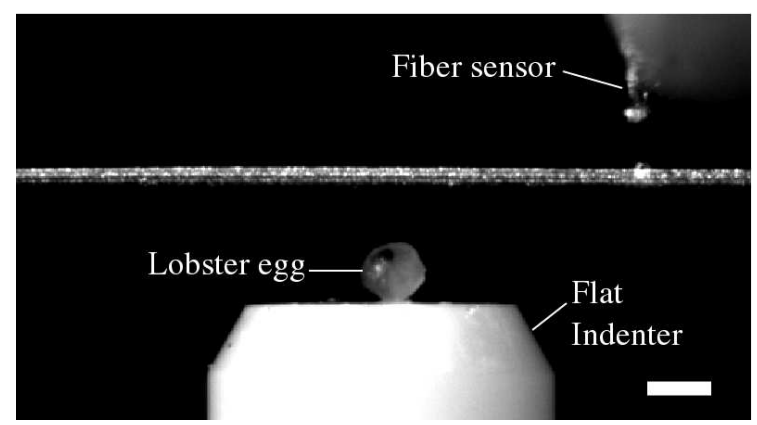

Figure 15. Side view of the slightly curved prototype for measuring the Young's modulus of a lobster egg. The optical fiber probe used for monitoring frequency shifts of the outer beams is visible in the upper right corner. Scale bar represents $500 \mu \mathrm{m}$.

The same protocol was repeated with a biological entity. Because embryos or oocytes could not be obtained easily, we used a lobster egg with a diameter of $500 \mu \mathrm{m}$ as a viable alternative (see Fig. 15). Linear frequency variations induced during the compression of the gel samples and the lobster egg are compared in Fig. 16(a).

To determine more precisely the elastic modulus of the lobster egg, slopes $S_{T 5}$ and $S_{T 7}$ indicated in Fig. 16(a) were used as single values plotted against the Young's modulus of the gels (see Fig. 16(b)). Since only the elastic properties of the materials were probed, a linear regression equation linking these two values could be determined $[42]$

$$
S_{\text {egg }}=0.02914 \times E_{\text {egg }}-0.2329
$$

where $S_{\text {egg }}$ is the slope of frequency variations measured for the lobster egg and $E_{\text {egg }}$ is its Young's modulus. For $S_{\text {egg }}=2.03 \mathrm{~Hz} / \mathrm{mN},(24)$ yields a Young's modulus of $78 \mathrm{kPa}$ for the lobster egg. Although no reference value has been found for lobster eggs in the literature by the authors, the Young's modulus obtained is in accordance with orders of magnitude usually reported for most living cells [43, 44, 45].

\section{Conclusion}

This paper has presented a new force sensitive structure exploiting a dynamic mode for probing the elastic modulus of living cells. A key feature of the triple beam configuration reported is the possibility to conduct measurements in liquids while keeping high dynamic performances. Another potential advantage is the possibility to adapt the structure's design for addressing both suspension and adherent cells. A first mesocale prototype has been characterized and calibrated with commercial gel samples. The 


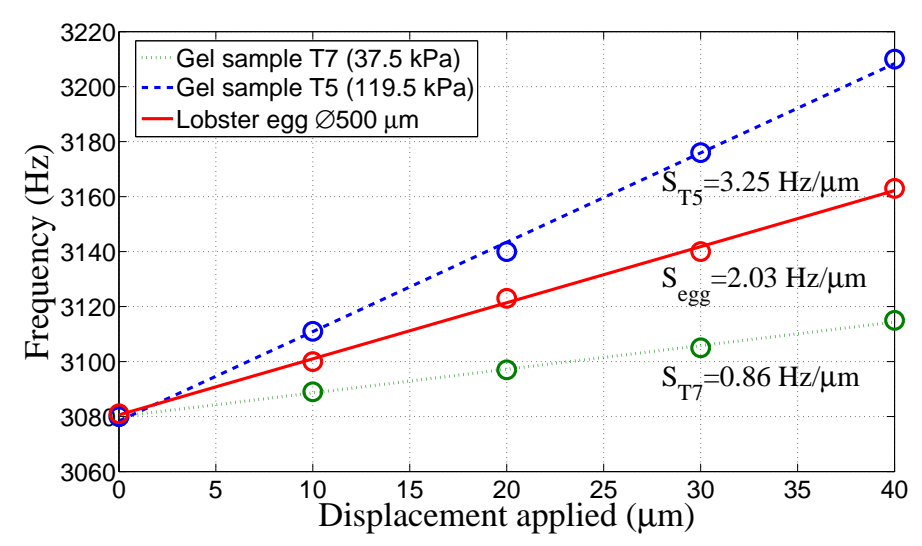

(a)

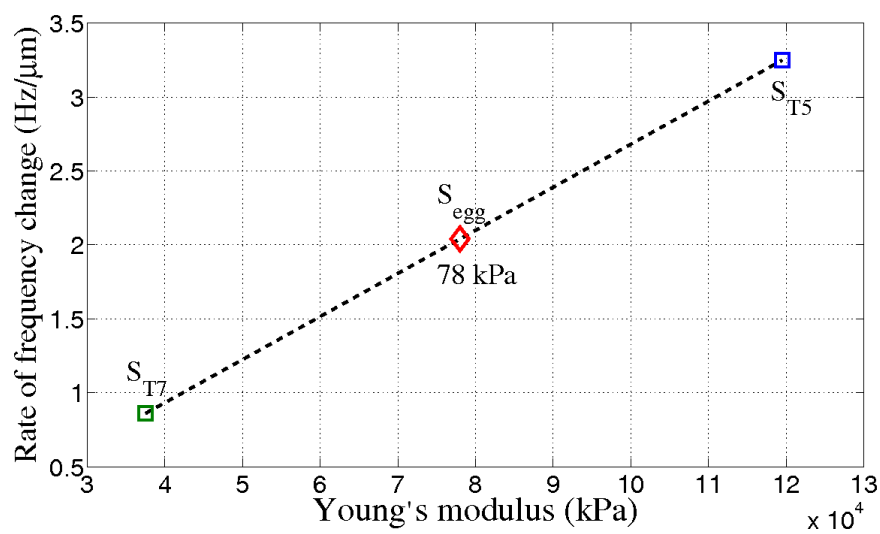

(b)

Figure 16. (a) Frequency variations measured during the compression of the commercial gel samples and the lobster egg; (b) Interpolation curve for extracting the Young's modulus of the lobster egg.

Young's modulus of a lobster egg has also been experimentally measured without the need of a descriptive model. Such an indirect technique might prove to be useful for diagnosis applications since relative changes or observation of tendencies may be sufficient to bring valuable information on the cell state.

Although performance characteristics of the prototype are encouraging, several challenges still need to be addressed. In particular, it remains presently difficult to obtain repeatable results due to our current clamping system. Likewise, maintaining the structure in a slightly curved position turns out to be a delicate task. A microfabricated version of the structure aimed at solving these problems is presently under investigation. The implementation of an open fluidic channel coupled to a simple positioning and/or trapping system is also ongoing. In the near future, tests with a larger number of cells will also be conducted to validate the possibility to implement the suspended structure in a workable platform. 


\section{References}

[1] Lee G Y H and Lim C T 2007 Trends Biotechnol. 25 111-8

[2] Cross S E, Jin Y S, Tondre J, Wong R, Rao J Y and Gimzewski J K 2008 Nanotechnology 19 384003

[3] Lekka M, Laidler P, Gil D, Lekki J, Stachura Z and Hrynkiewicz A Z 1999 Eur. Biophys. J. 28 $312-6$

[4] Zhao M, Srinivasan C, Burgess D J and Huey B D 2006 J. Mater. Res. 21 1906-12

[5] Li Q S, Lee G Y H, Ong C N and Lim C T 2008 Bioch. Bioph. Res. Commun. 374 609-13

[6] Cross S E, Jin Y S, Rao J Y and Gimzewski J K 2007 Nature Nanotech. 2 780-3

[7] Cross S E, Jin Y S, Lu Q Y, Rao J Y and Gimzewski J K 2011 Nanotechnology 22215101

[8] Maciaszek J L and Lykotrafitis G 2011 J. Biomech. 44 657-61

[9] Maciaszek J L, Andemariam B and Lykotrafitis G 2011 J. Strain Anal. Eng. Des. 44 368-79

[10] Carl P and Schillers H 2008 Pfügers Arch. 457 551-9

[11] Brandão M M, Fontes A, Barjas-Castro M L, Barbosa L C, Costa F F, Cesar C L and Saad S T O 2003 Eur. J. Haematol. 70 207-11

[12] Rotsch C and Radmacher M 2000 Biophys. J. 78 520-35

[13] Van Vliet K J, Bao G and Suresh S 2003 Acta Mater. 51 5881-905

[14] Huang H, Kamm R D and Lee R T 2004 Am. J. Physiol. - Cell Ph. 287 C1-11

[15] Norman J J, Mukundan V, Bernstein D and Pruitt B L 2008 Pediatr. Res. 63 576-83

[16] Hoffman B D and Crocker J C 2009 Annu. Rev. Biomed. Eng. 11 259-88

[17] Desmaële D, Boukallel M and Régnier S 2011 J. Biomech. 44 1433-46

[18] Lulevich V, Zink T, Chen H Y, Liu F T and Liu G y 2006 Langmuir 22 8151-5

[19] Waggoner P S and Craighead H G 2007 Lab Chip 7 1238-55

[20] Ayela C and Nicu L 2007 Sens. Actuators, B 123 860-8

[21] Johnson B and Mutharasan R 2011 Biosens. Bioelectron. 32 1-18

[22] Treyssède F 2007 J. Sound Vib. 307 295-311

[23] Ryu W, Huang Z, Park J S, Moseley J, Grossman A R, Fasching R J and Prinz F B 2008 Lab Chip 8 1460-7

[24] Ryu W, Bai S J, Park J S, Huang Z, Moseley J, Fabian T, Fasching R J, Grossman A R and Prinz F B 2010 Nano Lett. 10 1137-43

[25] Bai S J, Ryu W, Fasching R J, Grossman A R and Prinz F B 2011 Biotechnol. Lett. 33 1675-81

[26] Edwards C H 1994 The Mathematica Journal 4 48-52

[27] Venkateswara Rao G and Kanaka Raju K 2002 AIAA J. 40 1912-4

[28] Itoh H, Aoshima Y and Egawa T 2002 Jpn. J. Appl. Phys., Part $1413422-5$

[29] Hu Y C, Chang P Z and Chuang W C 2007 J. Micromech. and Microeng. 17 1870-6

[30] Morris D J, Yougsman J M, Anderson M J and Bahr D F 2008 Smart Mater. Struct. 17065021 (8pp)

[31] Lobontiu N 2007 Dynamics of Microelectromechanical Systems (Microsystems vol 17) (Springer) chap 4, pp 351-6

[32] Nicu L and Bergaud C 2004 J. Micromech. and Microeng. 14 727-36

[33] Seo J H and Brand O 2008 J. Microelectrom. Syst. 17 483-93

[34] Desmaële D, Boukallel M and Régnier S 2011 Proc. Int. Conf. on Intelligent Robots and Systems (San Francisco, CA, USA) (IEEE/RSJ) pp 913-8

[35] Dickinson S M 1980 J. Sound Vib. 68 507-14

[36] Yamaki N and Mori A 1980 J. Sound Vib. 71 333-46

[37] Elishakoff I, Birman V and Singer J 1985 Acta Mech. 55 191-202

[38] Kim C S and Dickinson S M 1986 J. Sound Vib. 104 170-5

[39] Treyssède F 2010 J. Sound Vib. 329 1536-52

[40] Liu K 2006 J. Phys. D: Appl. Phys. 39 R189

[41] Mathur A B, Collinsworth A M, Reichert W M, Kraus W E and Truskey G A 2001 J. Biomech. 
34 1545-53

[42] Murayama Y, Constantinou C E and Omata S 2004 J. Biomech. 37 67-72

[43] Bao G and Suresh S 2003 Nature Mater. 2 715-25

[44] Radmacher M 1997 IEEE Eng. Med. Biol. Mag. 16 47-57

[45] Kuznetsova T G, Starodubtseva M N, Yegorenkov N I, Chizhik S A and Zhdanov R I 2007 Micron 38 824-33 